\title{
Original
}

\section{Donante vivo renal. Experiencia de cirugia abierta y laparoscópica. Hospital La Paz}

\author{
Alfredo Aguilera Bazán, Manuel Pérez Utrilla, Ángel Alonso*, Enrique Jaureguizar Monereo**, \\ Luis Hidalgo Togores, Javier de la Peña Barthel \\ *Nefrología Pediátrica. **Urología Infantil. Hospital Universitario La Paz. Madrid.
}

\section{Resumen}

La extracción renal laparóscopica es una técnica escasamente implantada en nuestro país, debido principalmente a la dificultad técnica que supone y a la gran cantidad de donante cadáver que encontramos en nuestro medio. Presentamos y analizamos nuestra serie de donante vivo abierto y laparoscópico desde 1984.

Material y Métodos: Desde 1984 hasta 2007 se han realizado 84 extracciones renales de donante vivo; 64 por cirugía abierta, 20 laparoscópicas. El abordaje transperitoneal ha sido el elegido en el caso de la laparoscopia y la lumbotomía en la abierta.

Resultados: En la técnica abierta el tiempo quirúrgico medio es de $112 \mathrm{~min}$ (70-155), el tiempo medio de isquemia caliente es de 20 segundos (15-47) y la estancia media es de 4,8 días (3-9). En los casos realizados por laparoscopia, el tiempo quirúrgico medio es de 146 min (90-210), el tiempo de isquemia caliente es de 3 min y 15 seg $(2-3,25)$ y la estancia media es de 3,4 días (2-9).

Conclusiones: $\mathrm{El}$ abordaje laparoscópico es una técnica que no está exenta de riesgos y que no deben ser despreciados. Debe ser realizado por equipos con experiencia en cirugía renal laparoscópica. En lo referente al riñón obtenido de donante vivo, sin duda se trata de una magnífica solución para pacientes en estado de insuficiencia renal crónica. Es un recurso que deben ofrecer los principales hospitales de nuestro país.

Palabras clave: Donante vivo. Laparoscopia. Cirugía renal laparoscópica

\section{Renal live donor. Open and laparoscopic experience at La Paz Hospital}

\section{Abstract}

Laparoscopic live donor nephrectomy is a rare operation in our country because the complexity of the technique and the expansion of the cadaveric donor. We present our open and laparoscopic live donor nephrectomy from 1984.

Material and Methods: From 1984 to 2007 we have done 84 live donor nephrectomies; 64 open, 20 laparoscopic surgeries. The transperitoneal approach is preferred in laparoscopy and lumbotomy for the open surgery.

Results: In the open technique the operating time is 112min (70-155), ischaemia time 20 seconds (15-47) and postoperative hospital stay 4,8 days (3-9). Laparoscopic cases, the operating time is $146 \mathrm{~min}$ (90-210), ischaemia time 3 min 15 sec (2-3,25 $\mathrm{min})$ and postoperative hospital stay 3,4 days (2-9).

Conclusions: The laparoscopic live donor nephrectomy is a difficult and demanding technique. It should be done by experienced team in laparoscopic renal surgery. The kidney from a live donor is a very good alternative for the cronic renal failure. It should be offered in our main hospitals.

Keywords: Live donor. Laparoscopy. Laparoscopic renal.

$\mathrm{E}$ 1 periodo de tiempo más importante en el desarrollo de la Medicina y Cirugía en general, es sin duda el siglo XIX. El desarrollo de la anestesia comienza con la observación del dentista Horace Wells durante una exhibición con el llamado "gas hilarante" (protóxido de nitrógeno) durante la cual uno de los participantes no sufrió dolor alguno tras golpearse con un banco en la tibia. Sería un discípulo de éste, William Morton el que hiciera una primera demostración en la sala de operaciones del Massachussets General Hospital de Boston el 16 de Octubre de 1846, durante una cirugía de extirpación de tumor maxilar. El paciente, de unos 30 años, no sufrió dolor alguno. Acababa de nacer la anestesia por aspiración de gases. Sin duda, el otro gran 
avance de la época sería el desarrollo de la antisepsia hacia 1866. En este caso, el británico Joseph Lister basándose en los estudios de Louis Pasteur sobre la presencia de gérmenes en determinadas enfermedades y por otra parte al darse cuenta de la desaparición del olor pútrido de los campos de regadío de Carlisle con el empleo de ácido fénico, le llevó a desarrollar un método de antisepsia a base de este ácido. Su publicación en The Lancet en 1867 hizo que dicha técnica se extendiera mundialmente ${ }^{1}$.

Con estos dos avances del siglo XIX se había puesto la primera piedra de la cirugía moderna, que permitiría cirugías cada vez más prolongadas y complejas.

En 1933, el ucraniano Voronoy realiza el primer trasplante renal en humano. La paciente fallecería a las 48h de la intervención. Sería en 1947, en Boston, cuando se realizara el primer trasplante renal con éxito. Se trataba de una joven en coma urémico tras un shock séptico por un aborto complicado. Se le trasplantó un riñón de cadáver y fue implantado en la flexura del codo. En 1950 se realizará el primer trasplante renal con implantación intraabdominal a una mujer enferma de poliquistosis renal. Será en 1954, cuando se realice el primer trasplante renal con éxito absoluto, al trasplantar un riñón entre gemelos univitelinos ${ }^{2}$.

La realización del primer implante de donante vivo data de diciembre de 1952, en París (Hospital Necker), donde un hijo recibió el riñón de su madre. El injerto funcionó 20 días y el paciente falleció. Desde entonces, son numerosos los estudios realizados que demuestran la utilidad del riñón obtenido de un donante vivo frente al donante cadáver. El desarrollo de la laparoscopia renal en la década de los 90 llevó a los equipos quirúrgicos a plantearse la extracción laparoscópica de un riñón para donante vivo. En 1995, Ratner obtendría el primer riñón para trasplantar de esta manera ${ }^{3}$.

El donante vivo abre un abanico de posibilidades importantes, aportando unas claras ventajas para el receptor en lo que se refiere a mayor supervivencia del injerto o una mejor situación del receptor en el momento del implante, ya que llevará menos tiempo en lista de espera en diálisis o una mayor histocompatibilidad con el donante (suele ser entre familiares).

En España, actualmente, el 98\% de los trasplantes que se realizan proceden de donante cadáver y entre $1-2 \%$ son de donante vivo.
En este artículo, presentamos nuestra experiencia desde 1984 hasta nuestros días en la extracción de donante vivo por técnica abierta y laparoscópica.

\section{MATERIAL Y MÉTODOS}

Desde enero de 1984 hasta diciembre de 2007 hemos realizado 84 nefrectomías de donante vivo (64 abiertas, 20 laparoscópicas). En 73 ocasiones el receptor ha sido infantil y en 11 casos adulto. En 50 pacientes se eligió el riñón derecho y en 34 el izquierdo. En lo que se refiere a la relación entre los pacientes, en 19 ocasiones el padre era el donante, la hermana en 2 ocasiones, la madre en 62 casos y la mujer en 1 caso. La edad media es de 42 años. Todas las cirugías abiertas se han realizado por vía lumbar, mientras que las laparoscópicas el abordaje ha sido transperitoneal en 18 ocasiones y retroperitoenoscopia en 1 caso.

Todos los casos seleccionados tienen parentesco familiar entre ellos y han de someterse a una evaluación psicológica antes de la cirugía. Además, declararán ante un juez que se someten libremente a dicha intervención sin ningún tipo de coartación.

Como estudio preoperatorio especial de la anatomía vascular se realiza una angio RNM y una UIV para el estudio de la vía urinaria. El resto de estudios son los habituales de un preoperatorio.

Desde el punto de vista técnico, hasta el año 2005 se han realizado las extracciones por cirugía abierta (lumbotomía), dejando al donante el mejor riñón en caso de haber alguna diferencia entre ambos y eligiendo el derecho en caso de ser los dos iguales. Si alguno de ellos presenta multiplicidad de vasos del hilio renal y el otro no, se extraerá éste último. Con el desarrollo de la laparoscopia renal en nuestro servicio desde 2004, tras casi 70 intervenciones llevadas a cabo por el mismo cirujano, se realizó la primera extracción laparoscópica de donante vivo (Fig. 1). El criterio de selección del riñón a extraer es similar al que se emplea en la técnica abierta, pero en este caso se intenta, si las circunstancias lo permiten, que el riñón seleccionado sea el izquierdo, ya que la longitud vascular obtenida por esta técnica en el lado izquierdo es algo mayor que cuando se elige el lado derecho, sobre todo en la en lo referente a la vena.

La técnica laparoscópica normalmente empleada es el abordaje clásico transperitoneal con el paciente en decúbito lateral, ya descrito en múltiples artículos ${ }^{4,5}$ La disección y preparación del riñón 


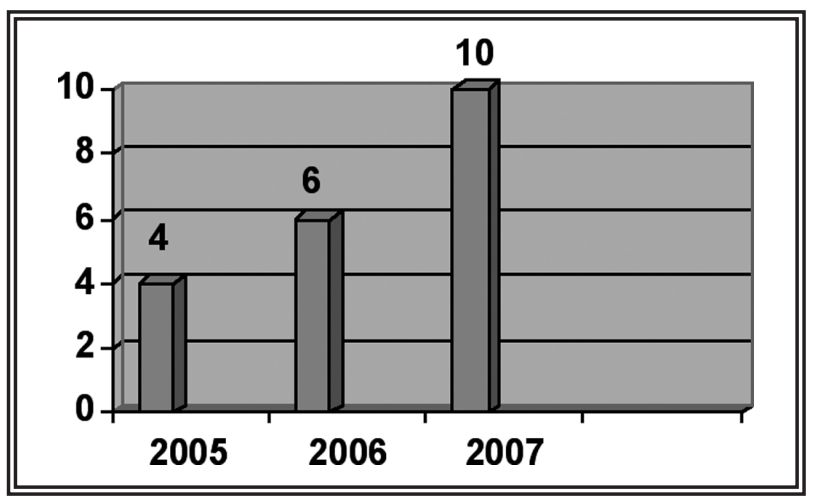

FIGURA 1. Extracción laparoscópica.

se realiza por laparoscopia pura y en el momento de la extracción se introduce la mano con el fin de que el tiempo de isquemia caliente sea menor. Al tratarse en muchas ocasiones de donantes jóvenes, la grasa perirrenal es escasa y debemos tener cuidado de no lesionar o decapsular el parénquima renal con la tracción ${ }^{6}$ El uréter lo disecamos respetando al máximo el tejido periureteral y la vena gonadal, con el fin de reducir los riesgos de lesión directa, por tracción o electrocauterio ${ }^{7,8}$. En el clampaje de la arteria renal, no ceñimos el clip al ostium, ya que hay descritos casos en la literatura de deslizamiento de los clips $^{9-11}$ Para la vena empleamos dos Hemolok XL, ya que las suturas mecánicas dejan menor longitud vascular. Según el modelo empleado, se disparan 4 o 6 tiras de grapas con el corte en el medio, de manera que posteriormente en la cirugía de banco, hay que recortar parte de la vena para quitar las 2 o 3 tiras de grapas que se quedan en el riñón. Una vez extraído, se realiza inmediatamente la perfusión con solución de Eurocollins en cirugía de banco.

\section{RESULTADOS}

En la técnica abierta el tiempo quirúrgico medio es de 112 min (70-155), el tiempo medio de isquemia caliente es de 20 segundos (15-47) y la estancia media es de 4,8 días (3-9). En los casos realizados por laparoscopia, el tiempo quirúrgico medio es de 146 min (90-210), el tiempo de isquemia caliente es de 3 min y 15 seg $(2-3,25)$ y la estancia media es de 3,4 días (2-9). En lo referente a las complicaciones, en la técnica abierta un $6 \%$ requirió transfusión intra o postoperatoria, 1 caso de trombosis femoral, 2 pacientes con hernias de la lumbotomía, 3 casos de parestesia persistente y 4 de atonía muscular. En las laparoscópicas, ningún caso requirió transfusión, 1 paciente presentó un hematoma de pared importante manejado conservadoramente, 1 absceso de herida quirúrgica y un caso de epigastralgia.En cuanto a los resultados funcionales del riñón trasplantado, se ha comparado la evolución de la función renal y la supervivencia del órgano con la serie global de trasplante de vivo con extracción quirúrgica. El tiempo total de isquemia fría fue similar en ambos grupos: ( $1,9 \pm 0,3$ horas). Durante el seguimiento del receptor no hubo diferencias significativas entre los dos grupos ni en la supervivencia a los 36 meses del paciente $(100 \%$ en la laparoscopia versus $98 \%$ en la quirúrgica) ni en la del injerto (90\% versus $93.21 \%$ ). En el análisis de riesgos múltiples de Cox el tipo de extracción tampoco fue determinante en la supervivencia del injerto. El grupo de laparoscopia necesita un mayor número de días $(9,56 \pm 2,3$ versus $4,72 \pm 0,57)$ en alcanzar la mínima cifra de creatinina, aunque el filtrado glomerular (expresado en $\mathrm{ml} / \mathrm{min} . / 1,73 \mathrm{~m}^{2}$ ) no es inferior a los 6 meses $(122 \pm 24$ versus $87 \pm 17$ $\left.\mathrm{ml} / \mathrm{min} . / 1,73 \mathrm{~m}^{2}\right)$; al año $(129 \pm 45$ versus $88 \pm 27$ $\left.\mathrm{ml} / \mathrm{min} . / 1,73 \mathrm{~m}^{2}\right) \mathrm{ni}$ a los dos años $(110 \pm 64$ versus $82 \pm 30 \mathrm{ml} / \mathrm{min}$. / $1,73 \mathrm{~m}^{2}$ ).

\section{DISCUSIÓN}

El trasplante renal es el mejor tratamiento para la insuficiencia renal terminal, ya que mejora la supervivencia y la calidad de vida del receptor respecto al paciente que se encuentra en diálisis. Por otra parte, España es el país del mundo con mayor número de órganos procedentes de donante cadáver al año ${ }^{12}$, de manera que entre el $98-99 \%$ de los trasplantes renales que se realizan, proceden de cadáver, mientras que en un $1-2 \%$ lo hacen de donante vivo.

Desde que en 1954 se realizara el primer implante de donante vivo con éxito, son múltiples los estudios que han demostrado las ventajas del riñón obtenido de esta fuente frente al procedente del cadáver; una mayor supervivencia a largo plazo (95\%-85\% a uno y tres años vs 90\%-70\% de cadáver), menor tasa de rechazo al tener una mejor histocompatibilidad y mejor función renal desde el inicio ya que el tiempo de isquemia es mucho menor ${ }^{13}$.

Actualmente, a pesar de ser España el primer país en donación de órganos procedentes de cadáver, sigue siendo la escasez de riñones la principal causa de muerte para los pacientes en lista de espera ${ }^{14}$. Sin duda la potenciación de la donación de vivo podría reducir de manera importante la lista de espera, ya 
que en nuestro país se encuentra claramente infrautilizada. En este sentido, nos podemos preguntar si la realización de la extracción por laparoscopia es un factor a tener en cuenta como estímulo positivo ${ }^{15,16 .}$ Para ello, debemos responder a varias preguntas, ya que no podemos ofrecer dicha técnica si el riñón obtenido no es útil para el implante; se trataría tan solo de un alarde quirúrgico. Desde que se realizara por primera vez en 1995, son varios los trabajos que han demostrado la funcionalidad de los riñones obtenidos de esta manera. Con un tiempo de isquemia caliente superior al de la cirugía abierta y un efecto deletéreo del neumoperitoneo sobre la función del riñón obtenido, debido sobre todo a la presión sobre el parénquima y la vena renal ${ }^{17,18,19}$, los riñones extraídos por laparoscopia pueden presentar un deterioro en la función renal en los primeros días, pero que se corrige posteriormente y es perfectamente comparable a los extraídos por técnica abierta al mes de la cirugía. En la literatura encontramos revisiones como la de Jacobs et $\mathrm{al}^{21}$ de 2004 donde describen la experiencia de la Universidad de Maryland con más de 700 casos o, también de 2004, la revisión de 44 estudios donde se comparan 2726 donantes por laparoscopia y 1537 por cirugía abierta y que refrendan la funcionalidad de los injertos así obtenidos ${ }^{22}$. Más recientemente, tenemos la revisión de un total de 69 trabajos publicada en 2007, donde la conclusión de la mayoría de los investigadores es la misma que la de los anteriores estudios en lo que a función se refiere, aunque el autor remarca la necesidad de que la cirugía laparoscópica se realice en centros con gran experiencia $^{23}$. En lo que se refiere a ese efecto negativo del neumoperitoneo sobre la función renal, se pueden tomar algunas medidas para minimizarlo, tales como la disección cuidadosa del hilio renal evitando las tracciones, hidratación abundante, uso de diuréticos osmóticos y no clipar el hilio renal hasta que no observemos una correcta diuresis por el uréter previamente cortado. Muchas veces, la imagen laparoscópica de una vena renal adecuadamente repleccionada es indicativo del grado de hidratación del paciente.

Una vez contrastada la utilidad de los riñones así extraídos, nos preguntamos qué es lo que aporta dicha técnica, ya que parece que al receptor no le afecta mucho que la extracción sea abierta o laparoscópica. Efectivamente, las claras ventajas de la técnica las vamos a encontrar en el donante; menor dolor postoperatorio, menos días de estancia hospitalaria, una más rápida incorporación a su vida normal y laboral y una mejor estética de las cicatrices $^{25}$. Tampoco debemos olvidar la morbilidad de la clásica lumbotomía, así como sus secuelas (infección, hernia, dolor crónico ${ }^{26,27}$ ).. Podemos plantearnos si estas ventajas pueden ser suficientemente importantes a la hora de que el donante se incline por aceptar la cirugía. Indudablemente la laparoscopia no va a hacer que todos los donantes a los que se la planteemos lo acepten, pero si hemos tenido casos en nuestra serie de pacientes cuyo trabajo autónomo no les permitía estar mucho tiempo de baja y la técnica mínimamente invasiva les ha hecho decidirse.

A medida que ha pasado el tiempo y con la popularización de la técnica, comienzan a aparecer en la literatura artículos que se plantean la seguridad de la técnica para el donante. Hay que tener en cuenta el tipo de cirugía que estamos planteando; sometemos a un paciente sano a una cirugía mayor, como es la nefrectomía, sin que vaya a obtener un beneficio directo. Además, el riñón obtenido no debe sufrir ningún daño y debe ser útil para trasplantar. En general, la gran mayoría de artículos y revisiones publicados llegan a la conclusión de que la laparoscopia es capaz de obtener riñones funcionantes con unas claras ventajas para el donante en lo que se refiere a convalecencia $^{21-24}$. Pero no debemos dejarnos deslumbrar por los resultados, ya que muchos de estos artículos nos describen la experiencia de centros de Estados Unidos donde el volumen anual de pacientes no tiene nada que ver con los europeos. En este sentido, el artículo de Kok et al de $2006^{28}$, muy crítico con la laparoscopia, nos describe la situación del noroeste de Europa, más cercana a nuestro entorno. Si bien es cierto que la conclusión a la que llega es que la laparoscopia es una técnica exigente que debe ser realizada por centros de experiencia, con lo cual estamos totalmente de acuerdo, lo que no quiere decir que dicha técnica sea de mayor riesgo para el donante.

En lo que se refiere a la vía de abordaje laparoscópica, el acceso transperitoneal tiene unas claras ventajas frente a la lumboscopia; mayor espacio de trabajo, más fácil orientación anatómica ${ }^{29,30}$. Aunque creemos que se debe conocer, ya que en algunas situaciones puede ser interesante la vía lumboscópica, no consideramos adecuado que la extracción de donante vivo sea el momento para ini- 
ciarse en dicho acceso. Por lo general, las series más largas y los principales artículos hacen referencia al abordaje transperitoneal, ya que suele ser una técnica más fácil y reproducible.

En cuanto a qué riñón podemos extraer por laparoscopia, el derecho o el izquierdo, lógicamente podemos obtener los dos, pero la dificultad aparece en el momento del implante. El riñón derecho, la longitud obtenida de la vena renal es claramente más corta que en el riñón izquierdo, lo que dificulta su implante y aumenta el riesgo de trombosis posterior ${ }^{31}$. En este sentido, el poder obtener una mayor longitud vascular se ve claramente limitada por el desarrollo de la tecnología; las suturas vasculares mecánicas son rectas, lo que dificulta poder ganar unos milímetros a la cava y además dejan 3 tiras de grapas que hay que recortar posteriormente de la vena renal. Serán bienvenidas modificaciones en dichos sistemas mecánicos, como el descrito por Bollens et $\mathrm{al}^{32}$, pero que todavía precisa del respaldo oficial de la empresa que lo fabrica. En lo que se refiere al clipaje de la arteria renal, que parecía claramente resuelto con el uso de clip plásticos Hem-o-lok, recientemente se han descrito deslizamiento de dichos clips, con el consiguiente accidente vascular, llegando la empresa que los fabrica (Wecks Closure Systems, Research Triangle Park, NC) a no recomendarlos para el donante vivo. Probablemente su deslizamiento se deba a la colocación excesivamente próxima al ostium de la arteria y la posterior sección de la misma apurando al máximo. Además, dos de los casos solamente se había colocado un solo clip, en lugar de $\operatorname{dos}^{9,10,11,34}$. Nosotros habitualmente colocamos dos clips plásticos, procurando dejar 1-2mm de distancia con el ostium y no apurando el corte al máximo.

Entre las complicaciones sufridas por la técnica laparoscópica, las más importantes son las pérdidas de 2 riñones en el postoperatorio inmediato. Uno de ellos, un implante pediátrico, tras una extracción correcta con un tiempo de isquemia caliente de 3 minutos hubo que revisarlo quirúrgicamente en las primeras horas ya que la evolución no era buena. Hay algunos artículos en la literatura que hacen referencia al deterioro de la función renal al implantar riñones de un adulto normotenso a un niño hipotenso ${ }^{35,36,37}$. La otra pérdida renal se produjo en un riñón extraído con un tiempo de isquemia de 3 minutos 30 segundos que se implantó en un receptor de 66 años con $\mathrm{BMI}>35$ y antecedentes de cirrosis biliar primaria. La anastomosis arterial fue dificultosa y hubo que revisarla quirúrgicamente también en el postoperatorio inmediato, produciéndose la pérdida final del riñón. En este caso, la indicación quirúrgica fue forzada por la familia, ya que el receptor no era un excelente candidato.

\section{CONCLUSIONES}

El donante vivo es una magnífica alternativa al donante cadáver, claramente infrautilizada en nuestro medio. Todo lo que suponga potenciar esta fuente de órganos resulta positivo. En este sentido, la laparoscopia realizada por equipos expertos parece ser lo suficientemente segura desde el punto de vista del donante y del riñón extraído. Pero en nuestro medio debemos tener en cuenta el número de pacientes mucho más bajo que en las series americanas, de manera que el aprendizaje y desarrollo del programa laparoscópico va a resultar más difícil, teniendo en cuenta que debe primar la seguridad del donante.

En lo que se refiere a la funcionalidad del riñón extraído por laparoscopia, la recuperación funcional del injerto obtenido se retrasa; sin embargo la función renal a los seis meses, al año y a los dos años del trasplante no es inferior a la de los pacientes con extracción quirúrgica convencional. La curva supervivencia del injerto y paciente y es similar con uno $\mathrm{u}$ otro procedimiento.

\section{REFERENCIAS}

1. Thorwald J. El siglo de los cirujanos. Ed Destino 1958. Pg 93153. ISBN: 9788423337248

2. Borel JF, Feurer C, Magne C, Schreier M. Effects of the new antilymphocytic cyclosporine A in animals. Inmunology 1977;32 (6): 1017-1025

3. Ratner LE, Ciseck LJ, Moore RG, Cigarroa FG, Kaufman HS, Kavoussi LR.Laparoscopic live donor nephrectomy. Transplantation 1995;60(9): 1047-1049.

4. El-Galley R, Hood N, Young CJ, Deierhoi M, Urban DA. Donor nephrectomy. A comparison of techniques and results of open, hand assisted and full laparoscopic nephrectomy. J Urol 2004; 171(1):40-43.

5. Handschin AE, Weber M, Demartines N, Clavien PA. Laparoscopic donor nephrectomy. Br J Surg 2003; 90(11):1323-1332.

6. Hammer C, Hawasli A, Meguid A and Oh H: Degloving of the renal capsule: a rare complication of laparoscopic live donor nephrectomy. J Laparoendosc Adv Surg Tech A 2006;16(4): 362-364.

7. Philosophe B, Kuo PC, Schweitzer EJ, Farney AC, Lim JW, Johnson LB, Jacobs S, Flowers JL, Cho ES, Bartlett ST. Laparoscopic versus open donor nephrectomy: comparing ureteral complications in the recipients and improving the laparoscopic technique. Transplantation 1999;68(4):497-502.

8. Fuller TF, Deger S, Buchler A, Roigas J, Schonberger B, Schnorr D, Tullmann M, Loening S, Giessing M. Ureteral Complications in the Renal Transplant Recipient after Laparoscopic Living Donor Nephrectomy. 2006;50(3):535-541. 
9. Maartense S, Heintjes RJ, Idu M, Bemelman FJ, Bemelman WA.: Renal artery clip dislodgement during hand-assisted laparoscopic living donor nephrectomy. Surg Endosc. 2003;17(11): 1851.

10. Hsu TH, Su LM, Ratner LE, Kavoussi LR. Reno vascular complications of laparoscopic donor nephrectomy. Urology 2002; 60(5):811-815.

11. Eswar C, Badillo FL: Vascular control of the renal pedicle using the hem-o-lok polymer ligating clip in 50 consecutive handassisted laparoscopic nephrectomies. J Endourol 2004;18(5): 459-461.

12. Memoria de la Organización Nacional de Trasplantes. 2007.

13. Cecka J. Results of more than 1000 recent living-unrelated donor transplants in the United States. Transplant Proc 1999; 31(1-2):234.

14. Modlin CS, Goldfarb DA, Novick AC. The use of expanded criteria cadaver and live donor kidneys for transplantation. Urol Clin North Am 2001;28(4):687-707.

15. Ratner LE, Hiller J, Sroka M et al. Laparoscopic live donor nephrectomy removes disincentives to live donation. Transplant Proc 1997; 29(8):3402-3403.

16. Schweitzer EJ, WilsonJ, Jacobs S, Machan CH, Philosophe B, Farney A. Colonna J, Jarrell BE, Bartlett ST. Increased rates of donation with laparoscopic donor nephrectomy. Ann Surg, 2000;232(3):392-400.

17. Razvi HA, Fields D, Vargas JC, Vaughan ED Jr, Vukasin A, Sosa RE..: Oliguria during laparoscopic surgery: evidence for direct renal parenchymal compression as an etiologic factor. J Endourol 1996;10:(1):1-4.

18. McDougall EM, Monk TG, Wolf JS Jr, Hicks M, Clayman RV, Gardner S, Humphrey PA, Sharp T, Martin K: The effect of prolonged pneumoperitoneum on renal function in an animal model. J Am Coll Surg, 1996;182(4):317-328.

19. Nguyen NT, Perez RV, Fleming N, Rivers R, Wolfe BM.: Effect of prolonged pneumoperitoneum on intraoperative urine output during laparoscopic gastric bypass. J Am Coll Surg 2002;195 (4):476-483.

20. Hazebroek EJ, de Bruin RW, Bouvy ND, van Duikeren S, Bonthuis F, Marquet RL et al: Short-term impact of carbon dioxide, helium, and gasless laparoscopic donor nephrectomy on renal function and histomorphology in donor and recipient. Surg Endosc 2002;16(2):245-251.

21. Jacobs S, Cho E, Foster C, Liao P, Bartlett S. Laparoscopic donor nephrectomy: The University of Maryland 6 years experience. J Urol 2004;171(1), 47-51.

22. Tooher R, Rao M, Scott D, Wall D, Francis D, Bridgewater, Maddern A. Systematic Review of Laparoscopic Live-Donor Nephrectomy. Transplantation 2004;78(3):404-414.

23. Shokeir AA. Open Versus Laparoscopic Live Donor Nephrectomy: A Focus on the Safety of Donors and the Need for a Donor Registry. J Urol 2007Vol;178(5):1860-186.

24. Alcaraz A, Rosales A, Guirado L, Díaz JM, Musquera M, Villavicencio H: Early experience of a living donor kidney transplant program. Eur Urol. 2006 sept; 50(3):542-547.

25. Ratner LE, Kavoussi LR, Sroka M, Hiller J, Weber R, Schulam PG, Montgomery R. Laparoscopic assisted live donor nephrectomy - a comparison with the open approach. Transplantation 1997;63(2):229-233.
26. Blohme I, Fehrman I, Norden G. Living donor nephrectomy: complication rates in 490 cases. Scand J Urol Nephrol 1992;26 (2): 149-153.

27. Dunn JF, Richie RE, MacDonell RC, et al. Living related kidney donors: a 14 year experience. Ann Surg 1986;203(6):637-643.

28. Kok N, Weimar W, Alwayn I, IJzermans J. The Current Practice of Live Donor Nephrectomy in Europe. Transplantation 2006; 82(7):892-897)

29. Desai M, Strzempkowski B, Matin SF, Steinberg AP, Meraney AM, Gill IS. Prospective randomized comparison of transperitoneal versus retroperitoneal laparoscopic radical nephretctomy. J Urol 2005; 173(1):38-41.

30. Abdelmaksoud A, Biyani CS, Bagheri F , Janetschek G. Laparoscopic approaches in urology. BJU International 2005;95(2): 244-249.

31. Mandal AK, Cohen C, Montgomery RA, Kavoussi LR and Ratner LE: Should the indications for laparoscopic live donor nephrectomy of the right kidney be the same as for the open procedure? Anomalous left renal vasculature is not a contraindication to laparoscopic left donor nephrectomy. Transplantation 2001;71(5):660-664.

32. Bollens R, Mikhaski D, Espinoza B, Rosenblatt A, Hoang AD, Abramowicz D, Donckier V, Schulman C. Laparoscopic Live Donor Right Nephrectomy: A New Technique to Maximize the Length of the Renal Vein Using a Modified Endo GIA Stapler. Eur urology 2007;51(5)(1326-1331.

33. Baldwin DD, Desai PJ, Baron PW, Berger KA, Maynes LJ, Robson $\mathrm{CH}$ et al. Control of the renal artery and vein with the nonabsorbable polymer ligating clip in hand-assisted laparoscopic donor nephrectomy. Transplantation 2005;80(3):310.

34. Kaushik M, Bagul A, Yates PJ, Elwell R, Nicholson ML. Comparison of Techniques of Vascular Control in Laparoscopic Donor Nephrectomy: The Leicester Experience. Transplantation Proceedings. 2006;38(10):3406-3408

35. Baqi N, Stock J, Lombrado SA, Geffner S and Roberti I: Impact of laparoscopic donor nephrectomy on allograft function in pediatric renal transplant recipients: a single-center report. Pediatr Transplant 2006;10(3):354-357.

36. Troppmann C, McBride MA, Baker TJ and Perez RV: Laparoscopic live donor nephrectomy: a risk factor for delayed function and rejection in pediatric kidney recipients? A UNOS analysis. Am J Transplant 2005;5(1):175-182.

37. Troppmann C, Pierce JL, Wiesmann KM, Butani L, Makker SP, McVicar JP et al. Early and late recipient graft function and donor outcome after laparoscopic vs open adult live donor nephrectomy for pediatric renal transplantation. Arch Surg 2002;137(8):908-915.

Correspondencia autor: Dr. Alfredo Aguilera Bazán Servicio de Urología. Hospital Universitario La Paz $\mathrm{P}^{\circ}$ de la Castellana, 261 - 28046 Madrid Tel.: 917277000

E-mail autor: aaguilera11@yahoo.es

Información artículo: Original - Traplante y Laparoscopia

Trabajo recibido: abril 2008

Trabajo aceptado: octubre 2008 